\title{
Evaluation of Clinical Co-Relation of Xi Point (Mid Point of Ramus) in Edentulous Patients with Reference to Occlusal Plane
}

\author{
A Meenakshi ${ }^{1}$, Nusrath Fatima ${ }^{2}$, Vinay Bharti ${ }^{2}$ and Sudarshan C Pujari ${ }^{3 *}$ \\ ${ }^{1}$ Professor, Department of Prosthodontics, Tamilnadu Govt. Dental College and Hospital Chennai, India \\ ${ }^{2}$ Post Graduate Student, Department of Prosthodontics, Tamilnadu Govt. Dental College and Hospital Chennai, India \\ ${ }^{3}$ M.S. Ramaiah University, Department of Conservative and Endodontics, Bangalore, India \\ *Corresponding Author: Sudarshan C Pujari, M.S. Ramaiah University, Department of Conservative and Endodontics, Bangalore, India.
}

Received: September 17, 2019; Published: October 21, 2019

DOI: $10.31080 /$ ASDS.2019.03.0673

\begin{abstract}
Introduction: In this clinical study the occlusal plane was established by two methods, in first method extra oral landmarks i.e. superior ala-tragal line is used as a reference for establishing the occlusal plane, and in second method intra oral landmarks, commissure of the mouth and upper third of retromolar pad were used as a reference to establish the occlusal plane. And later through digital cephalometric radiograph and DICOM software the proximity of the occlusal plane to Xi point is checked.

Materials and Methodology: Occlusal plane was traced up to the full width of ramus, parallelism of occlusal plane to ala-tragal line is verified and the distance between the Xi point to the occlusal plane was measured using DICOM software.

Results: The T- test comparison was done between control and study group. Occlusal plane established by method A method B was compared it shows the T- value of -15.470 and P- value of 0.0001 .

Conclusion: During the construction of complete denture the position of occlusal plane established by considering the superior alatragal line is similar to the position of OP of natural dentition.
\end{abstract}

Keywords: Ala-Tragal Line; Occlusal Plane; Xi Point

\section{Introduction}

The orientation of occlusal plane in natural dentition is one of the best examples of design that is present in nature. The conformity to this occlusal plane orientation by other parts of masticatory system is very distinct but it is often missed. It is very important to understand this interrelationship, because slight variation from this desired configuration can lead to occlusal instability.

According to GPT 8 [1] occlusal plane defined as "an average plane established by the incisal and occlusal surface of teeth. Generally it is not a plane but represents the planar means of the curvature". Another definition defines as "the surface of wax occlusal rims contoured to guide in the arrangement of denture teeth".

In completely edentulous condition the orientation of occlusal plane is lost due to the loss of natural teeth. The determination of correct occlusal plane inclination is necessary during the construction of complete denture in order to get the denture maximum stability and harmonious relation with masticatory system [2]. It is desirable to establish the occlusal plane in complete denture as it was present in natural dentetion [3].
Rickets [4] conducted a study on racial growth of mandible in 1955. During this study he investigated the relationship of occlusal plane in natural dentition to the ramus at different stages of growth of mandible. Results of this study showed that by extending the occlusal plane posteriorly to the full width of ramus, it was passing through the Xi point in subjects with good and stable occlusal relationship. So he concluded that in natural dentition for stable occlusion, the occlusal plane should pass through the Xi point.

By applying this concept in completely edentulous patient we can compare the different method of establishing the occlusal plane to find out in which method it passes through the Xi point. We selected Xi point as reference as it provides the fixed posterior reference point which can be located on cephalometric radiograph of edentulous mandible.

\section{Material and Methodology}

\section{Study design}

This clinical study was performed in dentate and edentate individuals to find out the correlation between the occlusal plane and Xi point(mid point of the ramus) (Figure 1). 


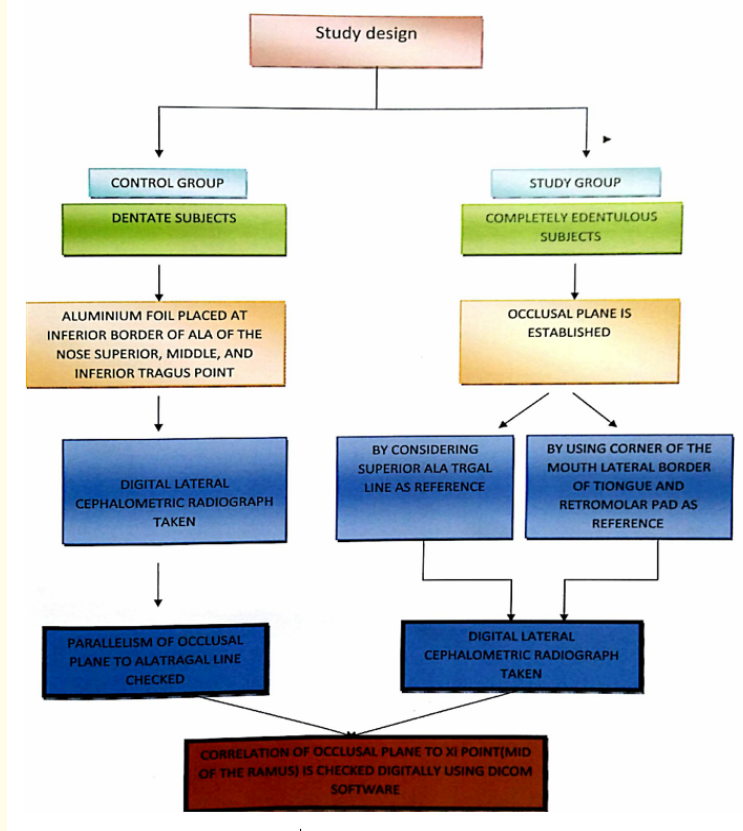

Figure 1: Study Design.

\section{Grouping of patients}

- Control Group: Individuals with full complement of teeth, Age group: 25-35years.

- Study Group: Completely edentulous individuals, Age group: 40-70years.

- Sample size: 20 (10 patients each for control and study groups).

\section{Methodology}

For control group

For taking the lateral cephalometric radiograph of the dentate patients superior, middle and inferior tragus points were identified and an aluminium foil of $1 \mathrm{~mm}$ radius was placed with the help of adhesive tape at the corresponding points. Later aluminium foil of $1 \mathrm{~mm}$ radius is placed with at the lower border of the ala of the nose with adhesive tape. This was done to get the radio opacity on the lateral cephalometric radiograph.

The patients Frankfort horizontal plane was oriented parallel to the floor. The mid-sagittal plane of the patient to the target was kept at $5 \mathrm{ft}$ distance and film to the midsagittal plane was $18 \mathrm{~cm}$ distance, central rays were coinciding with ear rod of the cephalostat.

Computerized digital cephalometric tracing to obtain values radiographically:

Radiographically obtained lateral cephalogram was traced using computerised DICOM (Digital imaging and communication in medicine) software. This software has features of performing tracing digitally. It allows zoom in for better visualisation of landmarks, inversion of image, to draw lines and measure angle between drawn lines and horizontal plane.

\section{To find out the co-relation of Xi point to occlusal plane}

Tracing of Xi point was done digitally by following

1. Construct four planes tangent to points $\mathrm{R}-1, \mathrm{R}-2, \mathrm{R}-3$ and $\mathrm{R}-4$ on the borders of the ramus

- R-1: Deepest point on the anterior border of the ramus,

- R-2: Located on the posterior border of the ramus opposite R-1,

- R-3: Deepest point of the sigmoid notch,

- R-4: Opposite R-3 on the inferior border of the mandible.

2. The constructed planes form a rectangle enclosing the ramus.

3. Xi point is located in the center of the rectangle at the intersection of the diagonals

After tracing of Xi point in dentate individuals, three tragus points, superior middle and inferior tragus points and lower border of ala of the nose were identified by locating radio opaque marks. Three ala-tragal lines were traced. Later tracing of OP was done from buccal cusp of premolars and molars excluding third molar to check the proximity of occlusal plane to Xi point and its parallelism with ala-tragal line using DICOM software.

\section{Jaw relation recording}

During jaw relation recording, occlusal plane is established by two different methods. In method A, anteriorly occlusal plane of maxillary occlusal rim is established by considering the esthetics [16]. i;e $2 \mathrm{~mm}$ visibility while speaking and posteriorly by considering the superior border of the tragus and lower border of the ala of the nose as reference plane [10] (Figure 2).

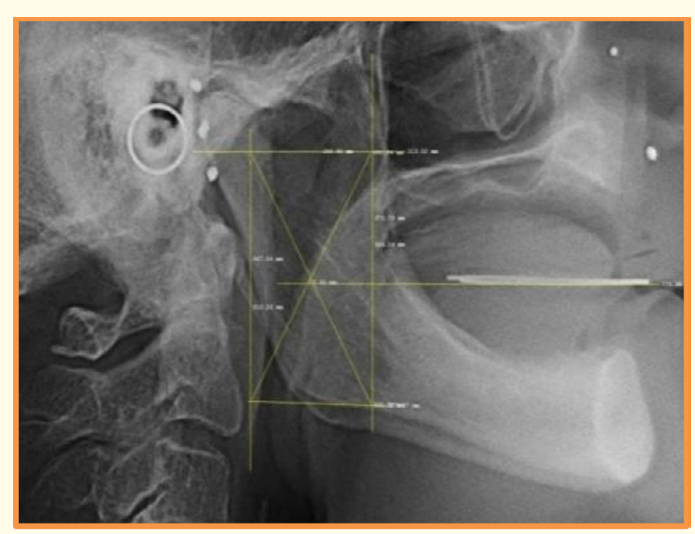

Figure 2: Lateral Cephalogram with Occlusal Plane Established by Method A. 
Parallelism of occlusal plane to superior ala-tragal line is verified clinically by using fox plane and scale. Later lower occlusal rim is adjusted according to maxillary rim at established vertical dimension of occlusion.

In method B (Figure 3), anteriorly occlusal plane of lower occlusal rim is established by considering the intra oral landmark $[8,12]$ commissure of the mouth, and posteriorly upper third of Retromolar pad. Later maxillary occlusal rim is adjusted according to plane of lower occlusal rim at established vertical dimension of occlusion.

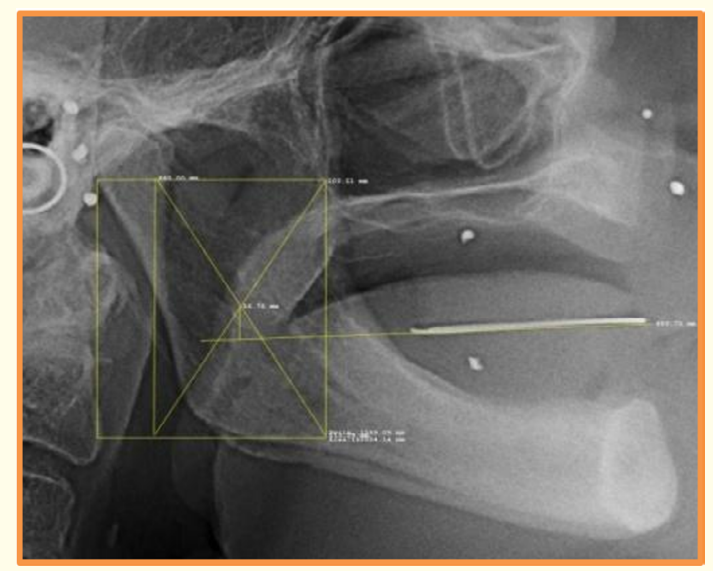

Figure 3: Lateral Cephalogram with Occlusal Plane Established by Method B.

Later, orthodontic stainless steel wire of desired length was cut. This was shaped according to the arch form and shape. This wire was placed on the established occlusal surface of the occlusal rims. Aluminium foil and orthodontic wire gives the radio opacity on the lateral cephalometric radiograph. Lateral cephalometric radiographs were taken at vertical dimension of occlusion.

Two lateral cephalometric radiographs were taken. The mid sagittal plane of the patient to the target was kept at $5 \mathrm{ft}$ distance and film to the midsagittal plane was $18 \mathrm{~cm}$ distance, central rays were coinciding with ear rod of the cephalostat.

Tracing of Xi point was done in similar manner and then tracing of occlusal plane established by method A and method B was done. Occlusal planes were traced up to the full width of ramus, and parallelism of occlusal plane of method A to superior ala-tragal line was verified and the distance between the Xi point and occlusal plane established by both methods were measured using DICOM software. Later tracing was done manually after the overlapping of two cephalograms to compare the two occlusal planes (Figure 4).

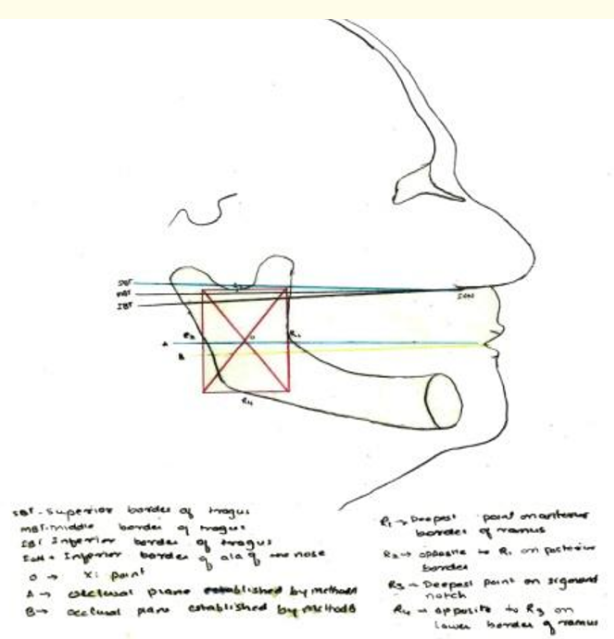

Figure 4: Tracing obtained after overlapping of two cephalograms to compare the two occlusal planes.

Results

From the values obtained in control group (Table 1) it is clear that difference in distance between occlusal plane and superior ala-tragal at anterior and posterior reference point is least and significant difference is present between occlusal plane to middle and inferior ala-tragal line, which indicates that superior ala-tragal line and occlusal plane are parallel to each other. And OP has close proximity to Xi point.

\begin{tabular}{|c|c|c|c|c|}
\hline Reading & N & Mean & $\begin{array}{c}\text { Std } \\
\text { Deviation }\end{array}$ & $\begin{array}{c}\text { Std error } \\
\text { of mean }\end{array}$ \\
\hline Control Group & 10 & 7.8 & 2.59678 & 0.82117 \\
\hline
\end{tabular}

Table 1: Shows the mean, standard deviation values and standard error of mean obtained by measuring the distance of occlusal plane from Xi point in dentate individuals.

After observing the above results in dentate individuals, superior tragus line is used as a reference line in establishing the occlusal plane in edentulous subjects.

Table 2 shows method A in which occlusal plane was established by considering the superior ala-tragal line as a reference plane shows the mean of 8.8(10 times of millimetre) with standard deviation and standard error of mean is 1.108 and 0.35 respectively. Values obtained in method A are very close to the values obtained in control group.

In method B in which occlusal plane was established by considering the commisure of mouth anteriorly and upper third of retromolar pad posteriorly shows the mean of 57.9(10 times of 


\begin{tabular}{|l|c|c|c|c|}
\hline Reading & N & Mean & Std Deviation & Std error of mean \\
\hline Method A & 10 & 8.8 & 1.10872 & 0.35061 \\
\hline Method B & 10 & 57.9 & 9.98921 & 3.15886 \\
\hline
\end{tabular}

Table 2: Shows the mean, standard deviation values and standard error of mean obtained by measuring the distance of occlusal plane from Xi point in edentate individuals.

millimetre) with standard deviation and standard error of mean is 9.9 and 3.15 respectively. Values obtained by method B are significantly greater than control group.

Table 3 shows the values obtained by measuring the distance between occlusal plane and Xi point. Comparison of method A and method B shows a significantly difference in values. There was no significant difference in values between control group and method $A$, and there was a significant difference in values of control group and method B.

\begin{tabular}{|l|c|c|c|}
\hline & & t-value & p-value \\
\hline Pair 1 & Method A-method B & -15.470 & 0.0001 \\
\hline Pair 2 & Method A-control & -1.07 & 0.302 \\
\hline Pair 3 & Method B-control & -15.359 & 0.0001 \\
\hline
\end{tabular}

Table 3: The pair t-test comparison between dentate and edentate group.

From the above results it is clear that the occlusal plane established by method A and method B were significantly different from each other with reference to Xi point. The OP established by method A was passing very close to Xi point as seen in dentate individuals.

\section{Discussion}

An analysis of cephalometric landmarks provides useful information on the craniofacial skeleton and the orientation of occlusal plane in dentulous and edentulous subjects. Generally cephalometric radiograph can be taken in both conventional method or digital method.

In this study we preferred digital lateral cephalogram as according to Sandler, direct digitization of the radiograph on monitor is more reproducible when compared with hand instruments tracing or digitization of tracing.

In our study after taking digital cephalogram images were converted into DICOM (Digital imaging and communication in medicine) format. This DICOM software was provided with the digital center. The tracing for lateral cephalogram was done on computer screen itself. This software provides option for image inversion, image magnification and helps us to draw lines connecting various landmarks and measurement of angles between these lines.

This cephalometric study was done to find out the correlation of Xi point to OP established by using two different methods.

For years researchers have been studying landmarks that can better guide the clinician in establishing the $\mathrm{OP}$.

Various methods used are,

- By considering extraoral landmarks such as ala-tragal line $[9,10,16,17]$ and

- By considering intraoral landmarks such as commissure of the mouth [12] and Retromolar pad [8], tongue position, buccinators groove [12] and parotid papilla.

Among extraoral landmarks, ala-tragal line is the more common reference point used.

Another most common method used during establishment of occlusal plane is by considering the intraoral landmarks. As supported by Ismail Y.H Bowman [8] where he recommended positioning of the second molar at the upper third of retromolar pad region.

Tracing showed that Occlusal plane established by method A and method B was passing below the Xi point, with a mean value of 8.8(10 times of millimeter) and 57.9 (10 times of millimeter) respectively.

The reason for variation of OP relation to $\mathrm{Xi}$ point in dentulous and edentulous group may be due to the change in anteroposterior dimensions of ramus after the loss of natural teeth which ultimately leads to the slight shift in the position of Xi point. This was supported by the findings of Donald H. Enlow, Ph.D., Henry J. Bianco, and Stephen Eklund [15], according to them unlike the child's mandible, the posterior border of the ramus is resorptive and the postero anterior dimension of the ramus (not the whole mandible) becomes reduced and narrowed in conjunction with resorption along the anterior border.

T-test comparison between the values obtained by measuring the distance between occlusal plane and Xi point in method A and method B showed a significant difference with $P$ value of 0.0001 .

The OP established by method B is not constant in all the individuals. In some individuals it was passing close to the $\mathrm{Xi}$ point with a value of 14.86 (10 times of millimeter) and in some individuals it was passing with posteriorly downward inclination in relation to Xi point with a value of 88.35 (10 times of millimeter). 
The results of this study showed that the OP established by method A has a close proximity to Xi point in comparison to OP established by method B.

Additionally in this study it was observed that the OP established by using intra oral landmarks showed the variable position in relation to Xi point so they should not be used as reliable reference landmarks whereas the OP established by use of superior border of the tragus showed more constant position in relation to Xi point so it is the most acceptable point to orient the occlusal plane.

\section{Summary and Conclusion}

The objective of this study is to find the correlation between $\mathrm{Xi}$ point and OP established by two methods.

OP was established by considering superior ala tragal line as reference in method A and in method B OP was established by considering commisure of mouth and upper third of the retromolar pad. Proximity of OP to Xi point is checked with the help of DICOM software.

The values were obtained and subjected to statistical analysis. From the analysis following conclusions were drawn

1. There was a significant difference in occlusal plane established by using superior ala-tragal line and commissure of mouth, retromolar pad in relation to $\mathrm{Xi}$ point.

2. The occlusal plane established by using superior ala-tragal line as reference was passing more closely to Xi point than the OP established by using commissure of mouth and retromolar pad as reference.

3. The OP established by using intraoral landmarks i.e. commissure of mouth and retromolar pad was not in constant position in relation to Xi point and so it is not a reliable landmarks.

Within the limitations of this study, the conclusion is that during the construction of complete denture the position of occlusal plane established by considering the superior ala-tragal line is similar to the position of OP of natural dentition. More research work needs to be carried out in large number of population to assess the validity of Xi point.

\section{Bibliography}

1. Glossary of prosthodontics terms. 8th edition.

2. Deogade SC., et al. "Lateral Cephalometric Radiographs: An Adjunct in Positioning the Occlusal Plane in Natural and Artificial Dentitions as Related to other Craniofacial Planes". Journal of Indian Academy of Oral Medicine and Radiology 23.4 (2011).
3. Sinobad D. "The position of the occlusal plane in dentulous subjects with various skeletal jaw-relationships". Journal of oral rehabilitation 15.5 (1998): 489-498.

4. Ricketts RM. "A principle of arcial growth of the mandible". The Angle Orthodontist 42 (1972): 368-386.

5. Shigli, K., et al. "Validity of soft tissue landmarks in determining the occlusal plane". Journal of Indian Prosthodontic Society 5.3 (2005).

6. Yasaki, M. "The height of the occlusion rim and the interocclusal distance". The Journal of Prosthetic Dentistry 11 (1961): 26-31.

7. Nagle RJ and SEARS YH. "Denture Prosthetics". 2nd Edn (1962): 134.

8. Ismail YH and Bowman JF. "Position of the occlusal plane in natural and artificial teeth". The Journal of prosthetic dentistry 20.5 (1968): 407-411.

9. Sharry JJ. "Complete denture prosthodontics". McGraw-Hill Companies (1974).

10. Fenn Liddelow. “Gimson”. Clinical Dental Prosthetics. 3rd edition. Wright London (1994).

11. Winkler S. "Essentials of complete denture prosthodontics". Saunders (1979): 183-190.

12. Lundquist DO and Luther WW. "Occlusal plane determination". The Journal of Prosthetic Dentistry 23.5 (1970): 489-498.

13. Javid NS. "A technique for determination of the occlusal plane". The Journal of Prosthetic Dentistry 31.4 (1974): 270-272.

14. L'Estrange PR and Vig PS. "A comparative study of the occlusal plane in dentulous and edentulous subjects". The Journal of Prosthetic Dentistry 33 (1975): 495-503.

15. Enlow DH., et al. "The remodeling of the edentulous mandible". The Journal of Prosthetic Dentistry 36.6 (1976): 685-693.

16. Spratley MH. "A simplified technique for determining the occlusal plane in full denture construction". Journal of oral rehabilitation 7 (1980): 31-33.

17. Neikerk Miller. "The ala tragus line in complete denture prosthodontics". The Journal of Prosthetic Dentistry 53 (1985): 67-68.

18. Sandler PJ. "Reproducibility of cephalometric measurements". Journal of Orthodontics 15.2 (1988): 105-110.

\section{Volume 3 Issue 11 November 2019 (C) All rights are reserved by Sudarshan C Pujari., et al.}

